\title{
Transgenic n-3 PUFAs Enrichment Regulates Related Physiological Activities by Affecting Nerves
}

\author{
Chao Song ${ }^{1}$, Jinyu Zhang ${ }^{1}$
} ${ }^{1}$ Department of Biochemistry and Molecular Biology, Medical College of Qingdao University, Qingdao Shandong,
China

\begin{abstract}
Obesity is generally defined as an excess of body fats and related to weight gain. The hypothalamic arcuate nucleus (ARC) is responsible for regulating peripheral signals that control food intake and energy balance. Uncoupling protein-1 (UCP-1) and UCP-2 are related-genes that adjust body weight. The aim of this study is to explore the effect of fat- 1 gene on body weight. We found that the weight/length ratios of fat- 1 transgenic mice were smaller than wild-type (WT) mice. We hypothesized that an increase in the levels of n-3 PUFAs might alter the expression of hypothalamic neuropeptide and lead to weight loss in fat-1 mice because fat-1 gene transforms n-6 polyunsaturated fatty acids (PUFAs) to n-3 PUFAs. Therefore, the appetite-regulating neuropeptides in the hypothalamic ARC, including neuropeptide Y (NPY), agouti-related peptides (AgRP), proopiomelanocortin (POMC), cocaine and amphetamine regulated transcript (CART), and ghrelin, were measured by Immunofluorescence. The mRNA levels of $U C P-1$ in brown adipose tissues and $U C P-2$ in white adipose tissues were measured by RT-PCR. The protein levels of UCP-2 in white adipose tissues also were measured by Western Blot. Compared with WT mice, the levels of CART, POMC and ghrelin increased, the levels of NPY and AgRP decreased, whereas the mRNA levels of UCP-1, UCP-2 increased, the protein levels of UCP-2 increased in fat-1 mice. The results indicated that the fat- 1 gene or n-3 PUFAs participate in inhibition of body weight by regulating the expressions of appetite neuropeptide and uncoupling protein.
\end{abstract}

Keywords: Hypothalamus, fat- 1 Gene, Neuropeptide, Body Weight

1. Introduction As the global obesity rate increases, obesity has become a major risk factor for several debilitating and deadly diseases in humans. However, the underlying mechanism of obesity is extremely complex and largely impacted by both environmental and genetic factors ${ }^{1}$. Obesity can be induced by the energy imbalance that is caused by any adverse effect on the regulation of appetite and energy metabolism in the body $y^{2,3}$. The hypothalamus, the control center of ingestive behavior and energy balance in humans, regulates the secretion of various neuropeptides. The hypothalamic arcuate nucleus (ARC), especially in its basal part, is the most important area. ARC plays an important role in appetite regulation and energy balance $^{4}$, as two groups of neurons control food intake and energy regulatory systems. The function of feeding inhibition recruits neurons which secrete proopiomelanocortin (POMC) and cocaine and amphetamine regulated transcript (CART) is to promote the energy metabolism in vivo and decrease appetite. On the contrary, the function of feeding stimulation recruits neurons that secrete neuropeptide $\mathrm{Y}(\mathrm{NPY})$ and agouti-related peptides (AgRP) is to promote appetite ${ }^{5}$. Ghrelin, a hormone synthesized by a special group of neurons, promotes ingestion, gastrointestinal movement, weight gain, and energy metabolism balance regulation ${ }^{6,}{ }^{7}$. Uncoupling protein-1 $(U C P-1)$ and $U C P-2$ play significant roles in energy metabolism because they both increase the energy consumption, especially in adipose tissues ${ }^{8}$.

As reported, n-3 PUFAs are critical in regulation of animal body weight and body fat around the abdomen $^{9,10,11}$. Generally speaking, mammals are unable to synthesize n-3 PUFAs, and can only take n-3 PUFAs from their diet. The functions of fat- 1 gene are to transform n-6 PUFAs into n-3 PUFAs in vivo ${ }^{12}$. Our previous studies showed that taking an n-3 PUFAs-rich diet a few months later, the rats' weight dropped significantly. ${ }^{13}$ Moreover, endogenous n-3 PUFAs in fat- 1 mice could reduce adipose tissue weight and control body weight ${ }^{14,15}$. However, the detailed mechanism that n-3 PUFAs affect body weight is unclear. Here, we investigated how endogenous n-3 PUFAs promote weight loss by regulating the hypothalamic expression of appetite neuropeptide and uncoupling proteins in a fat- 1 transgenic mouse model.

This article is published under the terms of the Creative Commons Attribution License 4.0

Author(s) retain the copyright of this article. Publication rights with Alkhaer Publications.

Published at: http://www.ijsciences.com/pub/issue/2019-03/

DOI: 10.18483/ijSci.1960; Online ISSN: 2305-3925; Print ISSN: 2410-4477 


\section{Materials and Methods}

\subsection{Grouping and feeding}

Fat-1 transgenic heterozygous mice (a kind gift from Professor Jingxuan Kang, Harvard Medical College and Jianbo Wan, University of Macau) were mated with C57BL/6 Wt mice. To induce the heterologous expression of Caenorhabditis elegans n-3 fatty acid desaturase in mice, Jingxuan Kang et al. modified the fat-1 gene by optimization of codon usage for mammalian cells and coupled it to a chicken beta-actin promoter (which allows high and broad expression of fat- 1 in mice). Then they microinjected the expression vector into fertilized eggs to produce transgenic mouse lines ${ }^{12}$. Polymerase chain reaction (PCR) was used to identify the offspring of FAT-1 transgenic mice. The male mice were then divided into a fat- 1 transgenic group and a WT group, with an average weight of $21 \mathrm{~g}$. All overweight mice $(>25 \mathrm{~g})$ or underweight mice $(<17 \mathrm{~g})$ were excluded. The six-week-old male heterozygous fat-1(+/-) mice and their WT littermates were bred in Qingdao University with access to standard rodent chow as described ${ }^{15,16}$ They were housed individually in environment-controlled conditions $\left(25 \pm 2{ }^{\circ} \mathrm{C}\right.$, light cycle from 06:00 to 18:00 and dark cycle from 18:00 to $06: 00)^{17}$ and allowed ad libitum access to food and water throughout the trials. After 8 weeks of cultivation, the animals were killed through cervical dislocation.

\subsection{Measurement of body weight and body length}

The body weight and body length of each animal were measured weekly. This study was approved and all procedures were performed in accordance with institutional guidelines of the Animal Care and Use Committee of Qingdao University.

\subsection{Immunofluorescence}

All groups of mice were transcardially perfused with $100 \mathrm{~mL}$ of $\mathrm{NS}$, followed by $400 \mathrm{~mL} \mathrm{4 \%} \mathrm{(w/v)}$ paraformaldehyde solution in $0.1 \mathrm{M}$ phosphate buffer (pH 7.4). The removal brain was fixed in $4 \%$ paraformaldehyde for $2 \mathrm{~h}$ and then transferred to $30 \%$ sucrose solution overnight at $4^{\circ} \mathrm{C}$. A series of $14 \mu \mathrm{m}$ brain coronal sections were cut on a freezing microtome (Kryostat 1720, Leica, Germany) ${ }^{20}$.

Sections were incubated with anti-NPY antibody (polyclonal; dilution, 1:250), anti-AgrP (polyclonal; dilution, 1:500), anti-POMC (polyclonal; dilution, 1:150), anti-CART (polyclonal; dilution, 1:150) and anti-Ghrelin (polyclonal; dilution, 1:500) at $4^{\circ} \mathrm{C}$ for $40 \mathrm{~h}$ and then incubated with a fluorochrome-labeled secondary antibody (Alexa Fluor 488-conjugated goat anti-rabbit IgG; dilution, 1:300) for $2 \mathrm{~h}$. Sections were then mounted with Citifluor. All fluorophores were visualized using a BX50 microscope and images were acquired using a DP50 digital camera
(Olympus, Tokyo, Japan). Stained brain sections were imaged using Zeiss LSM 510 laser scanning confocal microscope. All parameters were kept constant for quantification purposes. We used fluorescence intensity and cell counting, and positive cells were used to quantitatively measure cell numbers.

\subsection{Real Time PCR}

White and brown adipose tissue were homogenized using Trizol reagent (Invitrogen) kit and total RNA was isolated according to the manufacturer's protocol Total RNA $(2 \mu \mathrm{g})$ were reversely transcribed into cDNA using Transcriptor First Strand cDNA Synthesis Kit (Roche), and qRT-PCR was performed with Roche LightCycler 480 using SYBR Premix Ex Taq master mix (Roche). The specificity of qRT-PCR was confirmed by agarose gel electrophoresis and melting-curve analysis. A housekeeping gene (glyceraldehyde-3-phosphate dehydrogenase , GAPDH) was used as an internal standard. The primers used are listed in Table 1 . The relative fold changes were determined by the method of $2^{-} \Delta \Delta^{\mathrm{Ct}}$ as descript ${ }^{17}$. Usually, UCP-1 is mainly secreted by the brown adipose tissues and UCP-2 is mainly secreted by the white adipose tissues, so the mRNA of $U C P-2$ was extracted from WAT, the mRNA of $U C P-1$ from BAT.

\subsection{Western blotting}

Anti-UCP-2 and anti- $\beta$-actin were purchased from Bioss (China). For western blotting, lysates of white adipose tissue were prepared in RIPA buffer $(20 \mathrm{mM}$ Tris, pH 7.5, $5 \mathrm{ml}$ EDTA, $150 \mathrm{mM} \mathrm{NaCl}, 1 \% \mathrm{NP} 40$, $0.5 \%$ Na-deoxycholate, $0.025 \%$ SDS, $1 \mathrm{mM}$ Na-orthovanadate, $\quad 10 \mathrm{mM} \quad \mathrm{NaF}, \quad 25 \mu \mathrm{M}$ $\beta$-glycerophosphate) containing protease inhibitors (Roche, Indianapolis, IN). Adipose tissue lysates were prepared from $\sim 200 \mathrm{mg}$ of inguinal WAT or scapular BAT by homogenizing tissues in $1 \mathrm{ml}$ RIPA buffer. After centrifugation at $13,200 \times \mathrm{g}$ for $15 \mathrm{~min}$ at $4^{\circ} \mathrm{C}$, the supernatants were subjected to Western Blotting as described ${ }^{21}$. $\beta$-actin was used as a loading control.

\subsection{Statistical analyses}

Data were expressed as mean \pm standard deviation (SD). SPSS 18.0 software (SPSS, Inc., Chicago, IL) was utilized to conduct all the statistical analyses of the results. One-way analysis of variance (ANOVA) and Student's t-test were used for the results of the weights of adipose tissues, immunofluorescence and RT-qPCR statistical analysis. Analysis of body weight / height ratio by Two-ways ANOVA. The values were considered statistically significant at $\mathrm{P}<0.05$.

\section{Results}

\section{1. fat-1 gene decreased weight/length ratio.}

The fat-1 transgenic mouse pups at birth were 
essentially indistinguishable in size from WT mouse. As shown in Fig.1, the body weight/length ratio of fat- 1 transgenic group was significantly lower than that of WT group. There was a significant difference in body weight/length ratios between fat- 1 transgenic mice and WT mice in the 14th week of age (fat-1:2.258 $\pm 0.02, \mathrm{WT}: 2.376 \pm 0.04 \mathrm{~g} / \mathrm{cm}, \mathrm{n}=6$ animals per group, $\mathrm{P}<0.05$, Fig.1). Two groups of mice eat the same food, each mouse eat 6-8 $\mathrm{g}$ per day, no significant difference.

3.2. fat-1 gene changed the levels of appetite neuropeptides.

In order to better understand the causes that contribute to the differences observed in weight/length ratios between the WT and fat-1 animals, brain sections from both groups were examined by immunofluorescence. The results are shown in Fig.2: the protein expression of three genes, CART, POMC and ghrelin were significantly elevated in fat-1 transgenic mice relative to their respective WT counterparts. Whereas, NPY, AgRP levels were reduced in fat- 1 transgenic mice.

\subsection{Effect of fat-1 gene on mRNA level of UCP-1 and $U C P-2$}

To examine the effect of fat-1 gene on weight/length ratio between the two groups, RNA extracted from WAT and BAT were examined by RT-PCR. The results are shown in Fig.3: the mRNA expressions of $U C P-1, U C P-2$ were significantly elevated in fat-1 transgenic mice relative to their respective WT controls (Fig3.).

\subsection{Effect of fat-1 gene on protein level of UCP-2}

Protein extracted from WAT was examined by western blotting. According to Fig.4, the expression level of UCP-2 was significantly elevated in fat-1 transgenic mice relative to their respective WT controls (Fig4.).

\section{Discussion}

The hypothalamus is an important center responsible for the synthesis and release of neuropeptides that regulate feeding behaviors and energy balance. Located in the bottom of the third ventricle, ARC, which bulges in the center, is the excitatory and inhibitory neurons that affect appetite. WAT stores excessive energy as triglycerides, while BAT decreases energy storage by generating heat ${ }^{22}$. $U C P-1$ and $U C P-2$ induce the oxidation and ADP phosphorylation, decrease ATP production, and increase energy consumption and heat yield, eventually leading to weight loss $9,26,27$.

Here we found that the fat- 1 gene, which increases the endogenous $n-3$ PUFA levels by transforming $n-6$ PUFAs to n-3 PUFAs, obviously reduced weight/length ratios of mice. This result is in accordance with a previous report ${ }^{17}$. The fat- 1 and WT mice showed remarkable differences in the weight/length ratios. Therefore, we speculated that the existence of the fat- 1 gene was responsible for the differences in body weight, length, adipose and hematic parameters. Our data also shows that the expressions of CART, POMC and ghrelin are upregulated, while the expressions of NPY and AgRP are downregulated in the fat- 1 mice compared with the WT mice. The results are in line with our previous study ${ }^{18}$. In the study we measured the relative levels of CART, POMC, NPY, AgRP, ghrelin mRNA in the hypothalamus of fat-1 transgenic mice and WT mice ${ }^{18,19}$. Therefore, we think that these expression changes might be a partial mechanism underlying the weight loss in fat-1 mice. In addition, the increase of $U C P-1$ and $U C P-2$ is also a reason for body weight reduction in the fat- 1 mice compared with the WT mice (Fig. 4).

The discovery over the last years about the distinct hypothalamic distribution pattern of numerous novel neuropeptides has certainly complicated the hypothalamus ${ }^{23}$. Ghrelin is a newly-found orexigenic neuropeptide that improves appetite. These peptides are the key to better understand how the hypothalamus integrates and coordinates neural and hormonal inputs/signals into neuroendocrine, autonomic and behavioral responses. Generally, the appetite of animals can be promoted by the elevation in NPY and AgRP levels, but can be reduced by the increase in the POMC and CART levels ${ }^{24}$. Interestingly, ghrelin is highly expressed in the fat- 1 mice, which is contrary to the reduction of body weight. Ghrelin is considered as a compensatory mechanism that actively protects metabolic balance ${ }^{25}$ The function of ghrelin could be decreased by the reduction in NPY and AgRP levels ${ }^{8,19}$. Here, we speculate that the high levels of ghrelin neuropeptides in fat-1 mice are needed to maintain normal action and compensate for the low levels of NPY and AgRP. Nevertheless, the mRNA levels of $U C P-1$ and $U C P-2$ are upregulated in fat- 1 mice, which might be responsible for the body weight reduction.

In conclusion, fat- 1 gene could reduce body weight by modulating the hypothalamic expressions of appetite neuropeptides and UCP proteins in adipose. Nonetheless, to understand obesity and develop novel therapies, we need further studies to determine the precise molecular mechanisms how the fat-1 gene regulates body weight.

\section{Acknowledgements}

This work was supported by the National Natural Science Foundations of China (No. 30772000); Natural Science Foundations of Shandong Province (No.ZR2018MH009). 


\section{References}

1. Hayes M R. Scientific familial lessons in ingestive behavior research: 2016 Alan N.

2. Epsteinresearch award. J. Physiology \& behavior. 2017, 176:214-216. PMID: 28137426

3. Gale S.M., V.D. Castracane, and C.S. Mantzoros, Energy homeostasis, obesity and eating disorders: recent advances in endocrinology. J. Nutr, 2004. 134(2): p. 295-8. PMID: 14747663

4. Ferrario C R, Labouèbe G, Liu S, et al. Homeostasis Meets Motivation in the Battle to Control Food Intake. J. Journal of Neuroscience the Official Journal of the Society for Neuroscience. 2016, 36(45):11469-11481. PMID: 27911750

5. Cone R D, Cowley M A, Butler A A, et al. The arcuate nucleus as a conduit for diverse signals relevant to energy homeostasis. J. International Journal of Obesity \& Related Metabolic Disorders Journal of the International Association for the Study of Obesity. 2001, 25 Suppl 5(s182):S63-7. PMID: 11840218

6. Klein M O, Mackay H, Edwards A, et al. POMC and NPY mRNA expression during development is increased in rat offspring brain from mothers fed with a high fat diet. $\mathrm{J}$ International Journal of Developmental Neuroscience. 2017.03.004. 64:14-20. PMID: 28323039

7. Gillard L, Billiauws L, Stan-Iuga B, et al. Enhanced Ghrelin Levels and Hypothalamic Orexigenic AgRP and NPY Neuropeptide Expression in Models of Jejuno-Colonic Short Bowel Syndrome. J. Scientific Reports. 2016, 6:28345.PMID 27323884

8. R. Pandit, J. W. de Jong, L. J. M. J. Vanderschuren. Neurobiology of overeating and obesity: the role of melanocortins and beyond. European journal of pharmacology. 660 (2011) 28-42. PMID: 21295024

9. Park B G, Park Y S, Park J W, et al. Anti-obesity potential of enzymatic fragments of hyaluronan on high-fat diet-induced obesity in C57BL/6 mice. J. Biochemical \& Biophysical Research Communications. 2016, 473(1): 290-295. PMID: 27012203

10. Hill JO, Peters JC, LinD, et a1. Lipid accumulation and body fat distribution is influenced by type of dietary fat fed to rats. J. Int J Obes Relat Metab Disord. 1993, 17(4):223-226. PMID: 8387971

11. Huangx F, XinX, McLennanP, et a1. Role of fat amount and type in ameliorating Diet inducedobesity: insights at the levelof hypothalamie arcuate nucleus leptin receptor, neuropeptide $\mathrm{Y}$ and proopiomelanocortin mRNA expression. J. Diabetes Obes Metab. 2004, 6(1):35-44. PMID: 14686961

12. Ji S, Hardy R W, Wood P A. Transgenic Expression of n-3 Fatty Acid Desaturase (fat-1) in C57/BL6 Mice: Effects on Glucose Homeostasis and Body Weight. J. Journal of Cellular Biochemistry. 2009, 107(4):809. PMID: 19396841

13. Jing X. Kang. Fat-1 Transgenic Mice: A New Model for Omega-3 Research. Prostaglandins Leukot Essent Fatty Acid. 2007; 77(5-6): 263-267. PMID: 18042365

14. L.J. QIAO, Z. ZHENG, W.H. Ma. Effect of PUFA-containing diet on gut microbiota and fat metabolism-related genes in rats. Food Sci. 17 (2014) 231-235.
15. D.E. Cintra, E.R. Ropelle, J.C. Moraes. Unsaturated fatty acids revert diet-induced hypothalamic inflammation in obesity. J. PLoS One. 7 (2012) e30571. PMID: 22279596

16. P. J. White, P. L. Mitchell, M. Schwab. Transgenic $\omega-3$ PUFA enrichment alters morphology and gene expression profile in adipose tissue of obese mice: Potential role for protectins. Metabolism. 64 (2015) 666-676. PMID: 25726444

17. M. Goebel-Stengel, L. Wang. Central and peripheral expression and distribution of NUCB2/nesfatin-1. Curr. Pharm. Des. 19 (2013) 6935-6940. PMID: 23537079

18. Y. Avraham, J. Katzhendler, L. Vorobeiv. Novel acylethanolamide derivatives that modulate body weight through enhancement of hypothalamic pro-opiomelanocortin (POMC) and/or decreased neuropeptide Y (NPY). J. Med. Chem. 56 (2013) 1811-1829.

19. Shuangshuang Ma, Yinlin Ge, Xiaoying Gai, Meilan Xue, Ning Li, Jingxuan Kang, Jianbo Wan, Jinyu Zhang. Transgenic n-3 PUFAs enrichment leads to weight loss via modulating neuropeptides in hypothalamus. Neuroscience Letters, Volume 611. 12 January 2016, Pages 28-32, ISSN 0304-3940. PMID: 26610903

20. Cabral A, Portiansky E, Sánchezjaramillo E, et al. Ghrelin activates hypophysiotropic corticotropin-releasing factor neurons independently of the arcuate nucleus. J. Psychoneuroendocrinology. 2016, 67:27-39. PMID 26874559

21. Boysen B G. Book Review: The Rat Brain in Stereotaxic Coordinates. J. Veterinary Pathology. 2008, 45(4):597-598. Google

22. Hwee, D. T., Baehr, L. M., Philp, A., Baar, K. \& Bodine, S. C Maintenance of muscle mass and load-induced growth in Muscle RING Finger 1 null mice with age. Aging Cell. 2014, 13:92-101.PMID: 23941502

23. Zhou Xiaoshuang, Li Rongshan,Liu Xinyan, Wang Lihua,Hui Peng, Chan Lawrence, Saha Pradip K, Hu Zhaoyong. ROCK1 reduces mitochondrial content and irisin production in muscle suppressing adipocyte browning and impairing insulin sensitivity. J. Scientific reports. 2016;6: 29669. PMID: 27411515

24. Sousa-Ferreira L, Garrido M, Nascimento-Ferreira I Moderate long-term modulation of neuropeptide $\mathrm{Y}$ in hypothalamic arcuate nucleus induces energy balance alterations in adult rats. J. PLoS One. 2011; 6(7): e22333. PMID: 21799827

25. Santollo Jessica, Yao Dachun, Neal-Perry Genevieve, Etgen Anne M. Middle-aged female rats retain sensitivity to the anorexigenic effect of exogenous estradiol. J. Behavioural Brain Research. 2012 Jun 15;232(1):159-64. PMID: 22522024

26. L.J. QIAO, Z. ZHENG, W.H. Ma. Effect of PUFA-containing diet on gut microbiota and fat metabolism-related genes in rats. Food Sci. 17 (2014) 231-235.

27. Nicholls DG, Locke RM. Thermogeneic mechanisms in brown fat. Physol Rev. 1984,41:940-946. PMID: 6320232

28. Zhang Y, Proenca R, Mafei M. et al. Positional cloning of the mouse obese gene and its human homologue. Nature. 1994, 372: 425-431. PMID: 7984236 


\section{Figure Legends}

Fig.1. Body Weight/Length ratios of Fat-1 transgenic mice and wild-type mice. The body weight/length ratio of fat 1 group was significantly lower than that of WT group. Values are expressed as means \pm SEM ( $\mathrm{t}=10.40$, $P<0.05$ ). fat-1, Fat-1 transgenic group and WT, wild-type group. a means $P<0.05$ vs. WT. $\mathrm{n}=6$ animals per group.

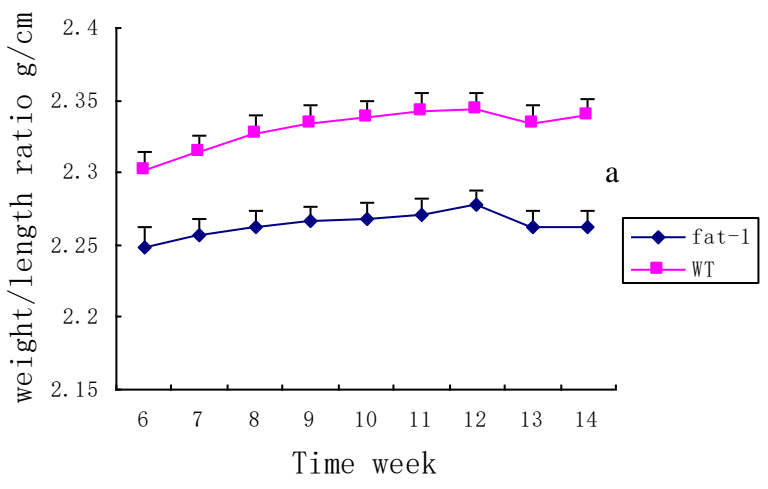

Fig.2. Representative immunofluorescence images highlights the expression of POMC, CART, NPY, AgRP and Ghrelin in neurons belonging to the hypothalamic arcuate nucleus. POMC (a), CART (b) and ghrelin were significantly elevated in fat- 1 mice than that of WT mice. On the contrary, NPY and AgRP levels were reduced in fat-1 mice. The negative control showed background staining (F, W). fat-1: Fat-1 transgenic group, WT: wild-type group. The statistical chart is used to count the positive cells quantitatively. Scale bar is $100 \mu \mathrm{m}$. Scale bar of the squares inset the images is $25 \mu \mathrm{m}$. Bar graphs compare POMC (A), CART (B), NPY (C), AgRP (D), and ghrelin (E) levels in fat-1 and WT mice. Values are expressed as means \pm SEM. fat-1: Fat-1 transgenic group, WT: wild-type group. a means $\mathrm{P}<0.05$ vs. WT, $\mathrm{n}=6$ animals per group.

a
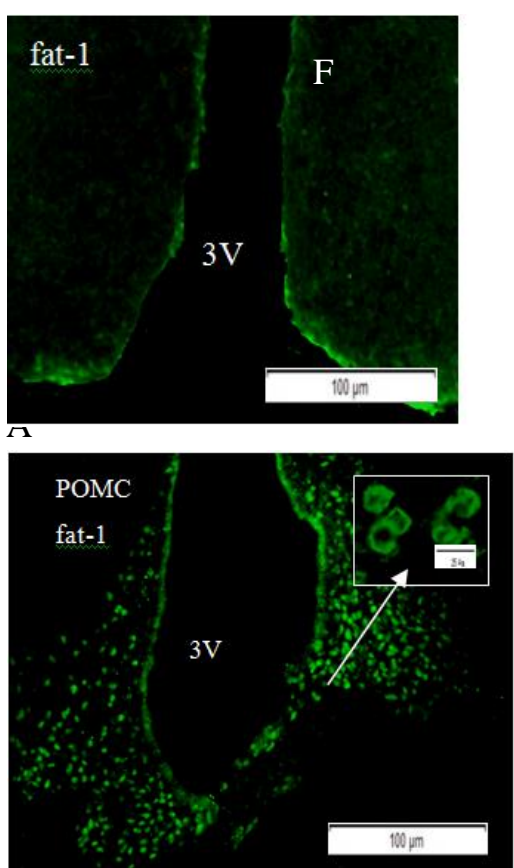

b
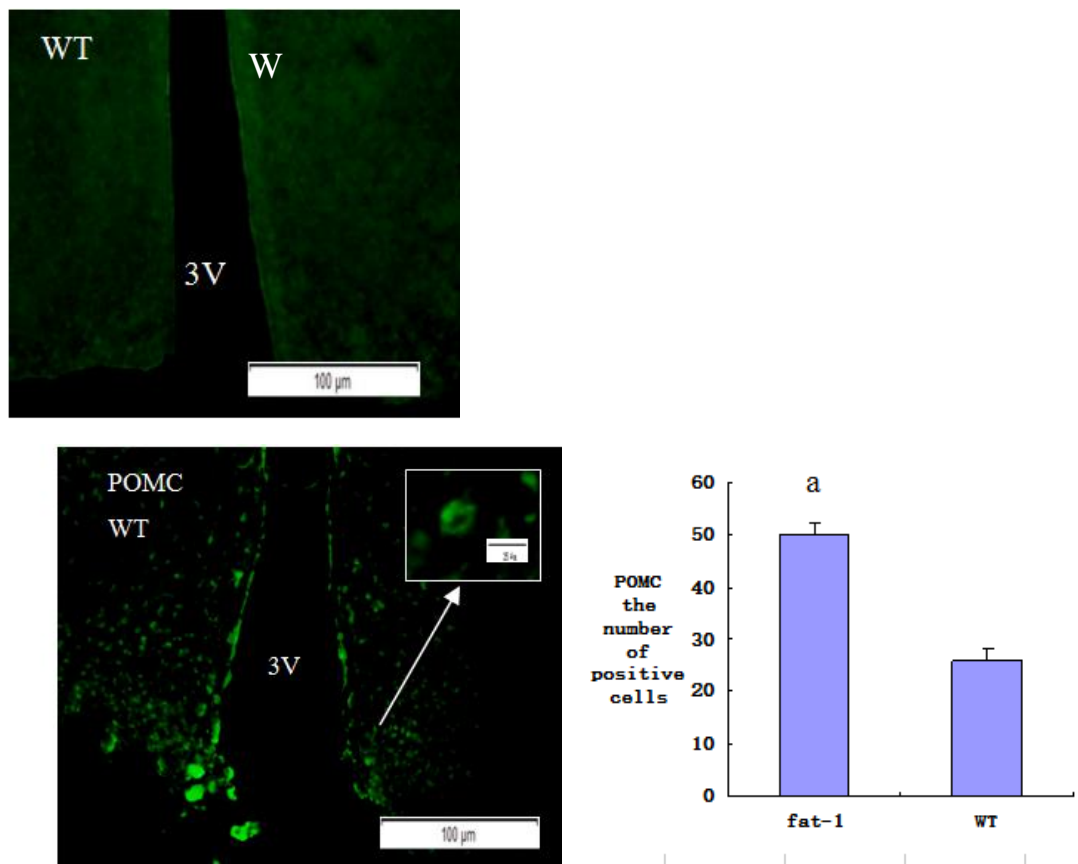

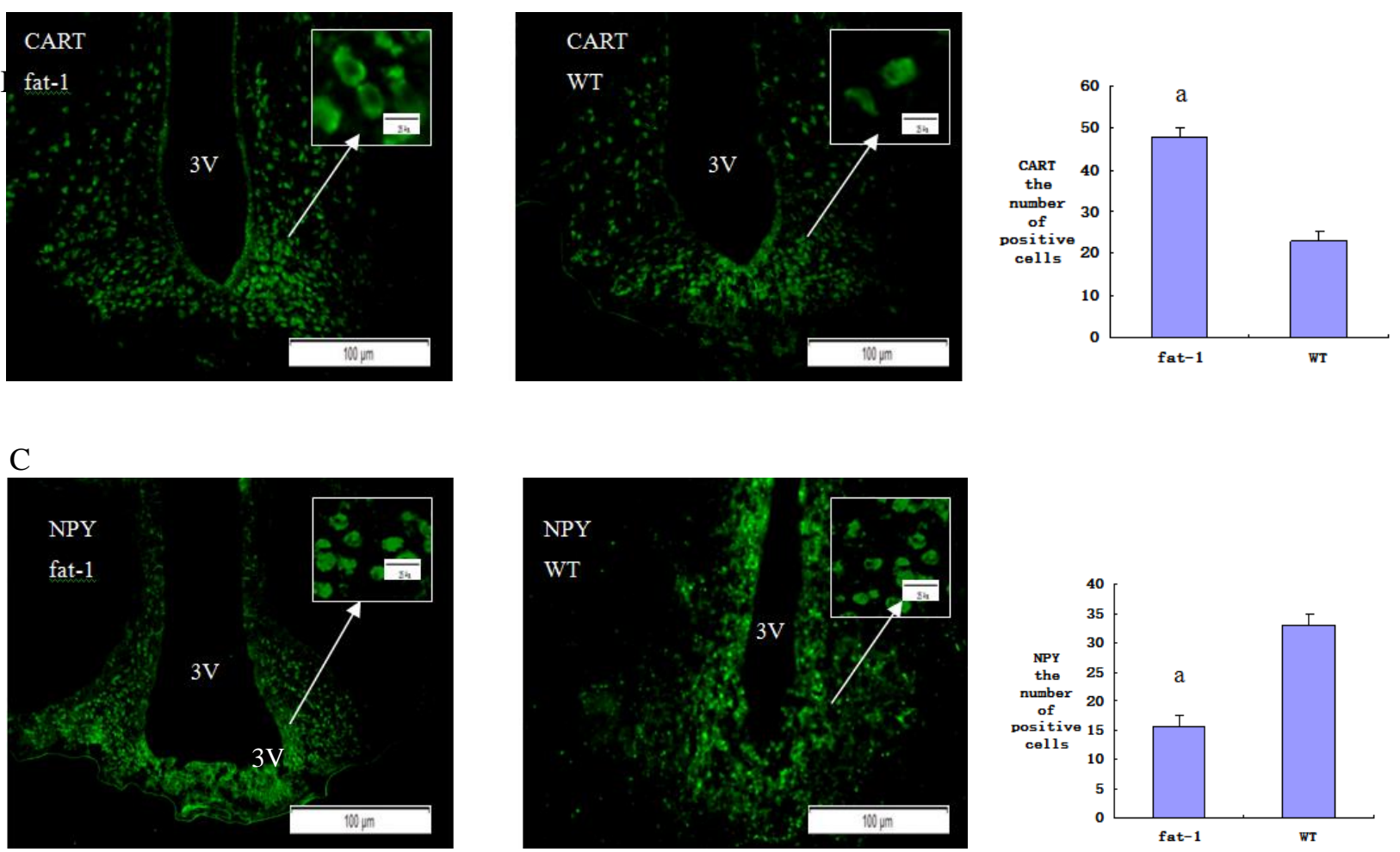

$\mathrm{D}$
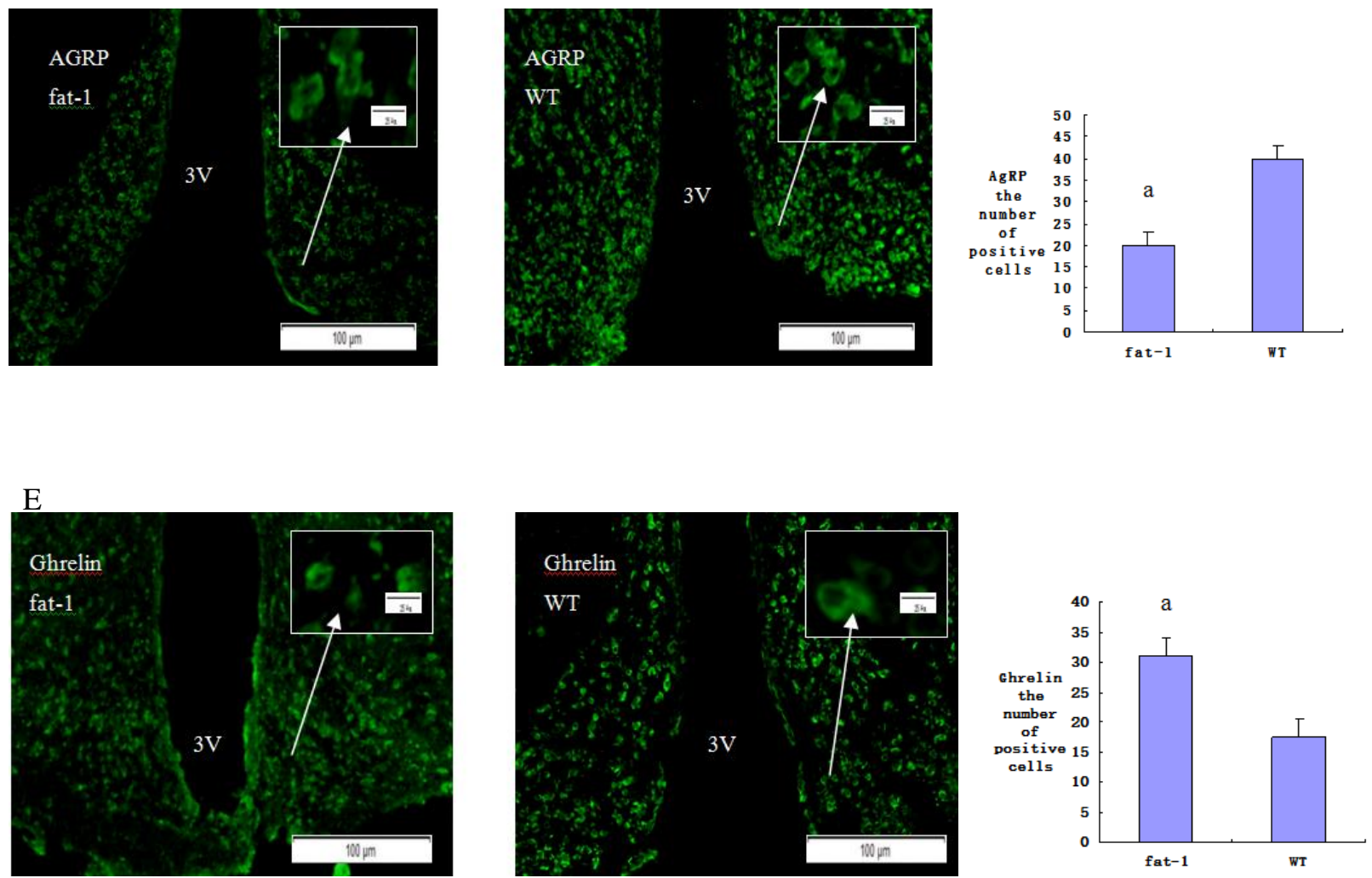
Fig.3. Comparisons of $U C P-1$ (A) and $U C P-2$ (B) mRNA in fat- 1 transgenic mice and wild-type mice. The mRNA expressions of $U C P-1$ (A) and UCP-2 (B) were significantly elevated in fat-1 mice compared to WT mice. Values are expressed as means \pm SEM. fat-1: Fat-1 transgenic group, WT: wild-type group. a means $\mathrm{P}<0.05$ vs. WT. $\mathrm{n}=6$ animals per group.
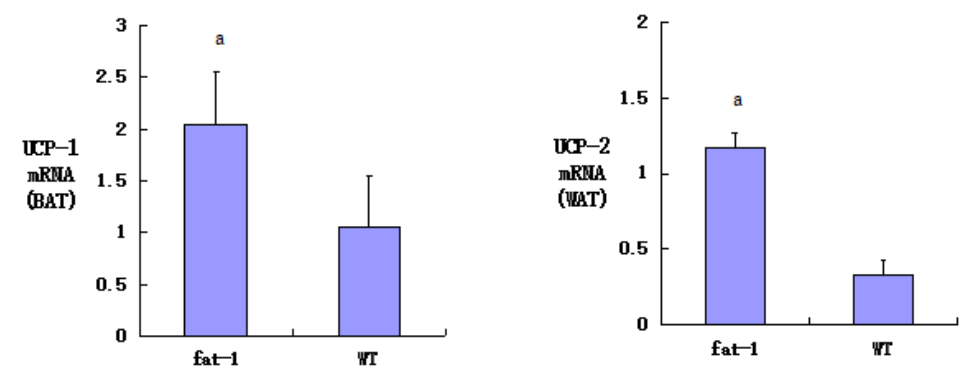

Fig.4. Comparisons of UCP-2 protein in fat-1 transgenic mice and wild-type mice. The protein expressions of UCP-2 were significantly elevated in fat-1 mice compared to WT mice. Values are expressed as means \pm SEM. fat-1: Fat-1 transgenic group, WT: wild-type group. a means $\mathrm{P}<0.05$ vs. WT. $\mathrm{n}=6$ animals per group.

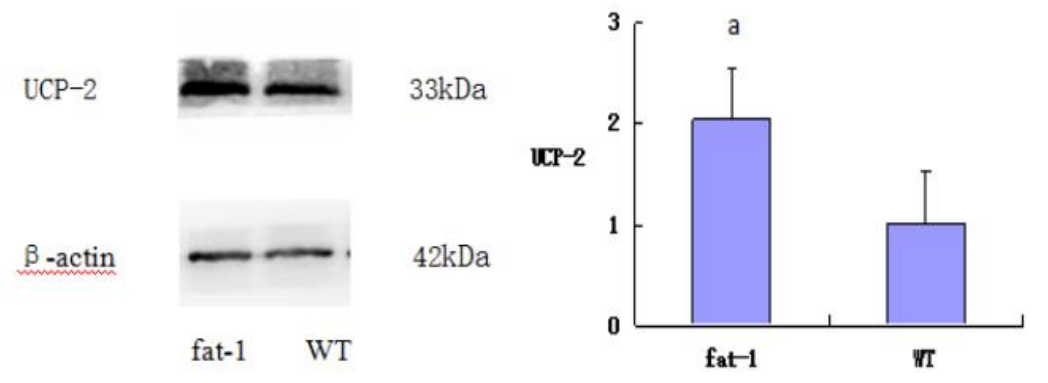

